\title{
Synthesis of 4,4'-(Arylmethylene)bis(1H-pyrazol-5-ols) Using Silica-bonded Ionic Liquid as Recyclable Catalyst
}

\author{
Mojtaba Baghernejad ${ }^{1} \&$ Khodabakhsh Niknam ${ }^{2}$ \\ ${ }^{1}$ Young Researchers Club, Gachsaran Branch, Islamic Azad University, Gachsaran, Iran \\ ${ }^{2}$ Department of Chemistry, Faculty of Sciences, Persian Gulf University, Bushehr 75169, Iran \\ Correspondence: Khodabakhsh Niknam, Department of Chemistry, Faculty of Sciences, Persian Gulf University, \\ Bushehr 75169, Iran. E-mail: khniknam@gmail.com
}

Received: February 27, $2012 \quad$ Accepted: March 22, $2012 \quad$ Online Published: May 27, 2012

doi:10.5539/ijc.v4n3p52

URL: http://dx.doi.org/10.5539/ijc.v4n3p52

\begin{abstract}
4,4'-(Arylmethylene)bis( $1 H$-pyrazol-5-ols) were synthesized in the presence of $N$-(3-silicapropyl)- $N$-methyl imidazolium hydrogen sulfate $\left([\mathrm{Sipmim}] \mathrm{HSO}_{4}\right)$ as a recyclable solid acid catalyst from the reaction between aldehydes and 3-methyl-1-phenyl-5-pyrazolone in refluxing ethanol. [Sipmim] $\mathrm{HSO}_{4}$ showed much the same efficiency when used in consecutive reaction runs.
\end{abstract}

Keywords: $N$-(3-Silicapropyl)- $N$-methyl imidazolium hydrogen sulfate, catalyst, bis(1H-pyrazol-5-ols), synthesis

\section{Introducation}

In the recent years, ionic liquids were used as solvents due to their particular properties, such as the ability to dissolve many organic and inorganic substances and undetectable vapor pressure (Wasserscheid \& Welton, 2007). In addition, Brønsted acidic task-specific ionic liquids (BAILs), such as those possessing $\mathrm{HSO}_{4}{ }^{-}$as a counter anion find a broad application in organic synthesis, acting as both solvents and catalysts. Keim and co-workers reported the synthesis of 1-butyl-3-methylimidazolium hydrogensulfate ([bmim] $\left.\mathrm{HSO}_{4}\right)\left(\mathrm{Keim}_{\mathrm{m}}\right.$ et al., 2000). In addition, in the year of $2002\left([\mathrm{bmim}] \mathrm{HSO}_{4}\right)$ was used as a catalyst in the Friedel-Crafts alkylation (Wasserscheid et al., 2002). The other applications of these acidic ionic liquids such as acetalization and thioacetalization of carbonyl compounds (Gupta et al., 2007), Fischer indole synthesis (Xu et al., 2007), acetylation of alcohols and phenols (Wang et al., 2008), preparation of azides from alcohols (Hajipour et al., 2009), selective nitration of phenols (Tajik et al., 2009), synthesis of 1,8-dioxo-octahydroxanthenes (Niknam \& Damya, 2009), formylation of alcohols (Niknam et al., 2009), synthesis of polysubstituted quinolines (Tajik et al., 2011), have been proceeded with very good yields and selectivities. Recently, immobilization of acidic ionic liquids on solid supports has been designed and it can offer important advantages in handling, separation and reuse procedures. Based on economic criteria, it is desirable to minimize the amount of ionic liquid utilized in a potential process. Immobilized acidic ionic liquids have been used as novel solid catalysts, e.g., for esterification, nitration reactions (Qiao et al., 2006), acetal formation (Sugimura et al., 2007), and Baeyer-Villiger reaction (Chrobok et al., 2009).

The pyrazolones and bis-pyrazolones were paid much attention for their various biological activities such as selective COX-2 inhibitory (Cho et al., 2004), antitumor (Park et al., 2005; Clark et al., 2004), cytokine inhibitors (Clark et al., 2005). Bis-pyrazolones can be used as antidepressant (Bailey et al., 1985), gastric secretion stimulatory (Rosiere \& Grossman, 1951), antibacterial (Mahajan et al., 1991) and antifilarial agents (Chauhan et al., 1993). Moreover, 4,4'-(arylmethylene)bis(1H-pyrazzol-5-ols) are applied as pesticides (Londershausen, 1996), fungicides (Singh \& Singh, 1991) and dyestuffs (Hamama, 2001).

In recent years, different reagents were applied for the synthesis of 4,4'-(arylmethylene)bis(3-methyl -1-phenyl-pyrazol-5-ols) derivatives, some of them including condensation reaction between arylaldehydes and two equivalents of 5-methyl-2-phenyl-2,4-dihydro-3H-pyrazol-3-one (Li et al., 1998; Singh \& Singh, 1984; Pavlov et al., 1998; Buzykin \& Lonshchakova, 1971; Wang et al., 2005; Elinson et al., 2008; Sujatha et al., 2009; Niknam et al., 2010; Niknam \& Mirzaee, 2011; Mosaddegh et al., 2010; Zang et al., 2011; Tayebi et al., 2011).

Herein, we prepared [Sipmim] $\mathrm{HSO}_{4}$ according to previously reported procedure (Chrobok et al., 2009) (Scheme 
1) and used as heterogeneous solid acid catalyst for the synthesis of 4,4'-alkylmethylene-bis(3-methyl-5pyrazolones).

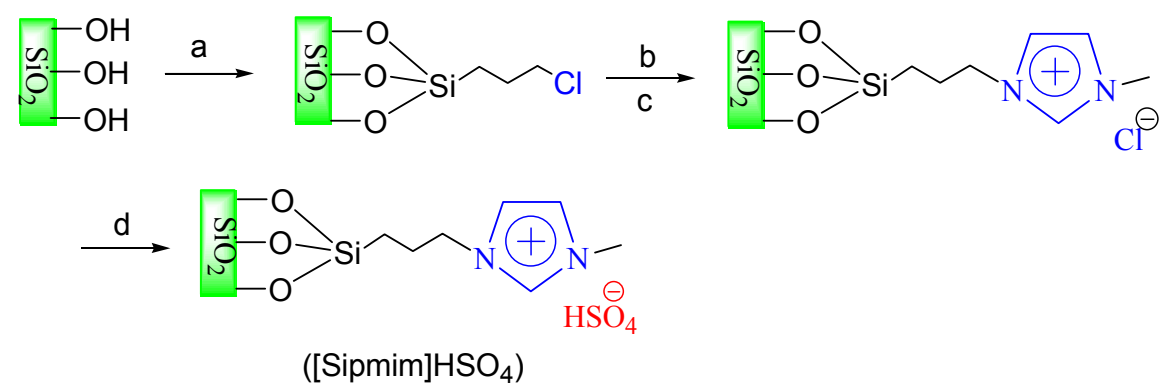

a) $(\mathrm{MeO})_{3} \mathrm{Si}-\left(\mathrm{CH}_{2}\right)_{3}-\mathrm{Cl}$, toluene(dry), $\mathrm{Et}_{3} \mathrm{~N}$, reflux, $48 \mathrm{~h}$; b) N-Methyl Imidazole, toluene (dry), $24 \mathrm{~h}$, reflux;

c) Dry under vaccum $\left(50^{\circ} \mathrm{C}\right.$ ), $4 \mathrm{~h}$; d) $\mathrm{CH}_{2} \mathrm{Cl}_{2}$ (dry, $\mathrm{H}_{2} \mathrm{SO}_{4}(97 \%), 48 \mathrm{~h}$, reflux

Scheme 1. Preparation of $N$-(3-silicapropyl)- $N$-methyl imidazolium hydrogen sulfate ([Sipmim] $\mathrm{HSO}_{4}$ )

\section{Method}

General: Chemicals were purchased from Merck and Fluka. IR spectra were run on a Shimadzu Infra Red Spectroscopy FT-IR-8000. The ${ }^{1} \mathrm{H}$ and ${ }^{13} \mathrm{C}$ NMR was run on Bruker Avance (DRX $500 \mathrm{MHz}$ and $400 \mathrm{MHz}$ ) instruments in DMSO- $\mathrm{d}_{6}$. Results are reported in ppm. Melting points were recorded on a SMP1 Melting Point apparatus in open capillary tubes and are uncorrected. Reaction progress was followed by TLC using silica gel SILG/UV 254 plates. 3-Chloropropyl silica, $N$-(3-silicapropyl)- $N$-methyl imidazolium chloride ([Sipmim]Cl), and $N$-(3-silicapropyl)- $N$-methyl imidazolium hydrogen sulfate ([Sipmim] $\mathrm{HSO}_{4}$ ) were prepared according to previously reported procedure (Chrobok et al., 2009).

\subsection{Catalyst Preparation}

\subsubsection{Preparation of $\mathrm{N}$-(3-silicapropyl)-N-methyl imidazolium Hydrogen Sulfate ([Sipmim] $\mathrm{HSO}_{4}$ )}

Into the three-necked round bottom flask equipped with stirrer, ice bath condenser, and thermometer $N$-(3-silicapropyl)- $N$-methyl imidazolium chloride ([Sipmim]Cl) $(3.0 \mathrm{~g})$ was suspended in dry $\mathrm{CH}_{2} \mathrm{Cl}_{2}(20 \mathrm{~mL})$. During vigorous stirring, concentrated $\mathrm{H}_{2} \mathrm{SO}_{4}(97 \%)(2.9 \mathrm{mmol})$ was added drop by drop at $0{ }^{\circ} \mathrm{C}$. Then the mixture was warm up to the room temperature, and was refluxing for $48 \mathrm{~h}$. When the formed $\mathrm{HCl}$ was completely distilled of the condenser the solution was cooled and the $\mathrm{CH}_{2} \mathrm{Cl}_{2}$ was removed under vacuum. To remove any water from the reaction mixture $10 \mathrm{~mL}$ of benzene was added to the crude ionic liquid and stirred for $3 \mathrm{~h}$ with magnetic stirrer at $50{ }^{\circ} \mathrm{C}$. Formed azeotrope was distilled of yielding [Sipmim] $\mathrm{HSO}_{4}$. Elemental analysis showed the $\mathrm{S}$ content to be $1.77 \%$. According to $\mathrm{S}$ content the number of $\mathrm{H}^{+}$sites of $[\mathrm{Sipmim}] \mathrm{HSO}_{4}$ is $0.55 \mathrm{mmol} / \mathrm{g}$.

\subsection{General Procedure for the Synthesis of 4,4'-(arylmethylene)bis(1H-pyrazol-5-ols)}

A round bottom flask contains $6 \mathrm{~mL}$ of ethanol was heated in an oil bath. When the temperature was reached to above $70{ }^{\circ} \mathrm{C}$, aromatic aldehyde $(1 \mathrm{mmol}), 5$-methyl-2-phenyl-2,4-dihydro-3 $\mathrm{H}$-pyrazol-3-one $(2 \mathrm{mmol})$ and [Sipmim] $\mathrm{HSO}_{4}\left(0.15 \mathrm{~g}\right.$, equal to $0.083 \mathrm{mmol}$ of $\left.\mathrm{H}^{+}\right)$were added to a flask and heated under reflux conditions. After completion of the reaction, as indicated by TLC, the reaction mixture was filtered. The remaining was washed with warm ethanol $(3 \times 30 \mathrm{~mL})$ in order to separate heterogeneous catalyst. After cooling the crude products were precipitated. The crude products were purified by recrystallization from ethanol $(95 \%)$. The recovered catalyst was dried and reused for subsequent runs.

All the products were characterized by comparison of their ${ }^{1} \mathrm{H}$ NMR and ${ }^{13} \mathrm{C}$ NMR spectroscopic data and their melting points with reported values (Li et al., 1998; Singh \& Singh, 1984; Wang et al., 2005; Elinson et al., 2008; Sujatha et al., 2009; Niknam et al., 2010; Niknam \& Mirzaee, 2011; Mosaddegh et al., 2010; Tayebi et al., 2011).

4,4'-(Phenylmethylene)bis(3-methyl-1-phenyl-1H-pyrazol-5-ol) (Table 2, entry 1). White cream solid; mp 170-172 ${ }^{\circ} \mathrm{C}$, [Lit.: (Wang et al., 2005) $171-172^{\circ} \mathrm{C}$ ]; $\delta_{\mathrm{H}}\left(300 \mathrm{MHz}\right.$; DMSO-d 6 ; $\left.\mathrm{Me}_{4} \mathrm{Si}\right) 2.32\left(6 \mathrm{H}, \mathrm{s}, 2 \times \mathrm{CH}_{3}\right), 4.96$ $(1 \mathrm{H}, \mathrm{s}, \mathrm{CH}), 7.17-7.27(7 \mathrm{H}, \mathrm{m}, \mathrm{Ar}), 7.44(4 \mathrm{H}, \mathrm{t}, J 7.7 \mathrm{Ar}), 7.71(4 \mathrm{H}, \mathrm{d}, J 7.9 \mathrm{Ar}), 13.96(2 \mathrm{H}, \mathrm{br}, \mathrm{OH}) . \delta_{\mathrm{C}}(75 \mathrm{MHz}$; DMSO-d $\mathrm{d}_{6}$; $\left.\mathrm{Me}_{4} \mathrm{Si}\right) 33.13,120.52,125.55,125.88,127.17,128.12,128.90,142.22,146.29$.

4,4'-[(4-Methylphenyl)methylene]bis(3-methyl-1-phenyl-1H-pyrazol-5-ol) (Table 2, entry 2). White solid; 
mp 202-204 ${ }^{\circ} \mathrm{C}$, [Lit.: (Li et al., 1998) $203{ }^{\circ} \mathrm{C}$ ]; $\delta_{\mathrm{H}}\left(300 \mathrm{MHz}\right.$; DMSO-d 6 ; $\left.\mathrm{Me}_{4} \mathrm{Si}\right) 2.24\left(3 \mathrm{H}, \mathrm{s}, \mathrm{CH}_{3}\right), 2.30(6 \mathrm{H}$, s, $\left.2 \times \mathrm{CH}_{3}\right), 4.90(1 \mathrm{H}, \mathrm{s}, \mathrm{CH}), 7.07(2 \mathrm{H}, \mathrm{d}, J 8.3 \mathrm{Ar}), 7.13(2 \mathrm{H}, \mathrm{d}, J 8.1 \mathrm{Ar}), 7.24(2 \mathrm{H}, \mathrm{t}, J 7.4 \mathrm{Ar}), 7.44(4 \mathrm{H}, \mathrm{t}, J 7.7$ Ar), $7.70(4 \mathrm{H}, \mathrm{d}, J 7.9 \mathrm{Ar}), 13.93(2 \mathrm{H}, \mathrm{br}, \mathrm{OH}) . \delta_{\mathrm{C}}\left(75 \mathrm{MHz}\right.$; DMSO-d $\left.{ }_{6} ; \mathrm{Me}_{4} \mathrm{Si}\right) 18.55,32.39,114.85,120.47$, $125.49,128.08,128.89,132.27,137.39,146.18,155.49$.

4,4'-[(4-Benzyloxyphenyl)methylene]bis(3-methyl-1-phenyl-1H-pyrazol-5-ol) (Table 2, entry 3): White solid; mp 210-212 ${ }^{\circ} \mathrm{C}$ [Lit.: (Li et al., 1998) $214{ }^{\circ} \mathrm{C}$ ]; $\delta_{\mathrm{H}}\left(400 \mathrm{MHz}\right.$, DMSO-d $\mathrm{d}_{6}$; $\left.\mathrm{Me}_{4} \mathrm{Si}\right) 2.32\left(6 \mathrm{H}, \mathrm{s}, 2 \times \mathrm{CH}_{3}\right), 4.91(1 \mathrm{H}$, s, CH), $5.06\left(2 \mathrm{H}, \mathrm{s}, \mathrm{CH}_{2} \mathrm{O}\right), 6.93(2 \mathrm{H}, \mathrm{d}, J 8.8 \mathrm{Ar}), 7.18(2 \mathrm{H}, \mathrm{d}, J 8.8 \mathrm{Ar}), 7.25(2 \mathrm{H}, \mathrm{t}, J 7.2 \mathrm{Ar}), 7.31(1 \mathrm{H}, \mathrm{t}, J 7.0$ Ar), $7.38(2 \mathrm{H}, \mathrm{t}, J 7.2 \mathrm{Ar}), 7.43-7.47(6 \mathrm{H}, \mathrm{m}, \mathrm{Ar}), 7.72(4 \mathrm{H}, \mathrm{d}, J 8.0 \mathrm{Ar}), 13.97(1 \mathrm{H}, \mathrm{br}, \mathrm{OH}) . \delta_{\mathrm{C}}(100 \mathrm{MHz}$, DMSO-d $\left.\mathrm{d}_{6} ; \mathrm{Me}_{4} \mathrm{Si}\right) 12.10,32.85,69.63,114.86,120.97,126.00,128.09,128.23,128.67,128.89,129.39,134.83$, 137.72, 146.69, 157.11. Anal. Calcd for $\mathrm{C}_{38} \mathrm{H}_{30} \mathrm{~N}_{4} \mathrm{O}_{3}$ : C, 75.26; H, 5.57; N, 10.33. Found: C, 75.09; H, 5.49; N, 10.18 .

4,4'-[(4-Acetamido- $N$-phenyl)methylene]bis(3-methyl-1-phenyl-1H-pyrazol-5-ol) (Table 2, entry 4): White cream solid; $\mathrm{mp} 251-253^{\circ} \mathrm{C} ; v_{\max }(\mathrm{KBr}) / \mathrm{cm}^{-1} 3405,3150,3025,2910,1660,1598,1570,1518,1485,1410,1370$, $1318,1275,1120,902,779,760,690 ; \delta_{\mathrm{H}}\left(400 \mathrm{MHz}, \mathrm{DMSO}-\mathrm{d}_{6} ; \mathrm{Me}_{4} \mathrm{Si}\right) 2.02 \quad\left(3 \mathrm{H}, \mathrm{s}, \mathrm{CH}_{3} \mathrm{CO}\right), 2.32(6 \mathrm{H}, \mathrm{s}, 2 \times$ $\left.\mathrm{CH}_{3}\right), 4.92(1 \mathrm{H}, \mathrm{s}, \mathrm{CH}), 7.18(2 \mathrm{H}, \mathrm{d}, J 8.4 \mathrm{Ar}), 7.25(2 \mathrm{H}, \mathrm{t}, J 7.2 \mathrm{Ar}), 7.43-7.47(6 \mathrm{H}, \mathrm{m}, \mathrm{Ar}), 7.72(4 \mathrm{H}, \mathrm{d}, J 7.6$ Ar), $9.88(1 \mathrm{H}, \mathrm{s}, \mathrm{NH}), 13.91(1 \mathrm{H}, \mathrm{br}, \mathrm{OH}) . \delta_{\mathrm{C}}\left(100 \mathrm{MHz}, \mathrm{DMSO}-\mathrm{d}_{6}\right.$; $\left.\mathrm{Me}_{4} \mathrm{Si}\right) 12.11,24.31,24.36,39.08,119.38$, $119.48,120.99,126.06,127.86,129.40,137.11,137.63,137.73,146.74,168.43,168.52$. Anal. Calcd for $\mathrm{C}_{29} \mathrm{H}_{27} \mathrm{~N}_{5} \mathrm{O}_{3}: \mathrm{C}, 70.57 ; \mathrm{H}, 5.51 ; \mathrm{N}, 14.19$. Found: C, 70.41; H, 5.42; N, 14.03 .

4,4'-[(5-Bromo-2-hydroxy-phenyl)methylene]bis(3-methyl-1-phenyl-1H-pyrazol-5-ol) (Table 2, entry 5): White cream solid; $\mathrm{mp} 266-268{ }^{\circ} \mathrm{C} ; v_{\max }(\mathrm{KBr}) / \mathrm{cm}^{-1} 3405,3080,2910,1620,1598,1570,1495,1405,1375$, $1275,1102,899,805,790,745,690 ; \delta_{\mathrm{H}}\left(400 \mathrm{MHz}, \mathrm{DMSO}_{6}\right.$; $\left.\mathrm{Me}_{4} \mathrm{Si}\right) 2.30\left(6 \mathrm{H}, \mathrm{s}, 2 \times \mathrm{CH}_{3}\right), 5.13(1 \mathrm{H}, \mathrm{s}, \mathrm{CH})$, $6.74(1 \mathrm{H}, \mathrm{d}, J 8.4 \mathrm{Ar}), 7.17(1 \mathrm{H}, \mathrm{d}, J 6.8 \mathrm{Ar}), 7.25-7.28(2 \mathrm{H}, \mathrm{m}, \mathrm{Ar}), 7.46(4 \mathrm{H}, \mathrm{t}, J 7.4 \mathrm{Ar}), 7.66-7.72(5 \mathrm{H}, \mathrm{m}, \mathrm{Ar})$. $\delta_{\mathrm{C}}\left(100 \mathrm{MHz}, \mathrm{DMSO}-\mathrm{d}_{6} ; \mathrm{Me}_{4} \mathrm{Si}\right) 12.19,27.91,110.28,117.40,121.14,126.21,129.43,130.06,131.61,146.74$, 153.87. Anal. Calcd for $\mathrm{C}_{27} \mathrm{H}_{23} \mathrm{BrN}_{4} \mathrm{O}_{3}$ : C, 61.03; H, 4.36; Br, 15.04; N, 10.54. Found: $\mathrm{C}, 60.84 ; \mathrm{H}, 4.29$; N, 10.38 .

4,4'-[(2-Bromophenyl)methylene]bis(3-methyl-1-phenyl-1H-pyrazol-5-ol) (Table 2, entry 6). White cream solid; mp 198-200 ${ }^{\circ} \mathrm{C}$, [Lit.: (Tayebi et al., 2011) $198-200{ }^{\circ} \mathrm{C}$ ]; $v_{\max }(\mathrm{KBr}) / \mathrm{cm}^{-1} 3440,3060,2920,1605,1560$, $1495,1395,1365,1300,830,744,690 ; \delta_{\mathrm{H}}\left(500 \mathrm{MHz}\right.$; DMSO-d 6 ; $\left.\mathrm{Me}_{4} \mathrm{Si}\right) 2.31\left(6 \mathrm{H}, \mathrm{s}, 2 \times \mathrm{CH}_{3}\right), 5.10(1 \mathrm{H}, \mathrm{s}, \mathrm{CH})$ $7.13(1 \mathrm{H}, \mathrm{t}, J 7.3 \mathrm{Ar}), 7.23(2 \mathrm{H}, \mathrm{t}, J 7.0 \mathrm{Ar}), 7.33(1 \mathrm{H}, \mathrm{t}, J 7.3 \mathrm{Ar}), 7.42(4 \mathrm{H}, \mathrm{t}, J 7.9 \mathrm{Ar}), 7.56(1 \mathrm{H}, \mathrm{d}, J 7.8 \mathrm{Ar})$, $7.71(4 \mathrm{H}, \mathrm{d}, J 7.9 \mathrm{Ar}), 7.86(1 \mathrm{H}, \mathrm{m}, \mathrm{Ar}), 12.68(1 \mathrm{H}, \mathrm{br}, \mathrm{OH}), 13.92(1 \mathrm{H}, \mathrm{s}, \mathrm{OH}) . \delta_{\mathrm{C}}\left(125 \mathrm{MHz} ; \mathrm{DMSO}_{6} \mathrm{~d}_{6} ; \mathrm{Me}_{4} \mathrm{Si}\right)$ $13.08,35.21,121.49,123.54,126.43,128.30,129.16,129.76,131.44,133.64,138.21,146.78$. Elemental analysis: for $\mathrm{C}_{27} \mathrm{H}_{23} \mathrm{BrN}_{4} \mathrm{O}_{2}$ : C, 62.92; H, 4.50; Br, 15.50; N, 10.87. Found: C, 62.74; H, 4.54; N, 10.70 .

4,4'-[(4-Chlorophenyl)methylene]bis(3-methyl-1-phenyl-1H-pyrazol-5-ol) (Table 2, entry 7). White solid; mp 215-217 ${ }^{\circ} \mathrm{C}$, [Lit.: (Li et al., 1998) $210{ }^{\circ} \mathrm{C}$ ]; $\delta_{\mathrm{H}}\left(300 \mathrm{MHz}\right.$; DMSO-d ${ }_{6}$; $\left.\mathrm{Me}_{4} \mathrm{Si}\right) 2.30\left(6 \mathrm{H}, \mathrm{s}, 2 \times \mathrm{CH}_{3}\right), 4.98(1 \mathrm{H}$, s, CH) 7.22-7.28 (4H, m, Ar), $7.35(2 \mathrm{H}, \mathrm{d}, J 8.5 \mathrm{Ar}), 7.44(4 \mathrm{H}, \mathrm{t}, J 7.9 \mathrm{Ar}), 7.71(4 \mathrm{H}, \mathrm{d}, J 7.9 \mathrm{Ar}), 13.90(2 \mathrm{H}, \mathrm{br}$, $\mathrm{OH}) . \delta_{\mathrm{C}}\left(75 \mathrm{MHz}\right.$; DMSO-d ${ }_{6}$; $\left.\mathrm{Me}_{4} \mathrm{Si}\right) 32.56,120.54,125.62,128.00,128.90,129.13,130.56,137.18,141.14$, 146.23 .

4,4'-[(2-Chlorophenyl)methylene]bis(3-methyl-1-phenyl-1H-pyrazol-5-ol) (Table 2, entry 8). White solid; mp 235-237 ${ }^{\circ} \mathrm{C}$, [Lit.: (Wang et al., 2005) $236-237^{\circ} \mathrm{C}$ ]; $\delta_{\mathrm{H}}\left(400 \mathrm{MHz}\right.$; DMSO-d 6 ; $\left.\mathrm{Me}_{4} \mathrm{Si}\right) 2.29\left(6 \mathrm{H}, \mathrm{s}, 2 \times \mathrm{CH}_{3}\right.$ ), 5.14 (1H, s, CH), 7.22-7.33 (4H, m, Ar), $7.40(1 \mathrm{H}, \mathrm{d}, J 7.8 \mathrm{Ar}), 7.44$ (4H, t, $J 7.6 \mathrm{Ar}), 7.70(4 \mathrm{H}, \mathrm{d}, J 7.6 \mathrm{Ar}), 7.80$ $(1 \mathrm{H}, \mathrm{d}, J 7.1 \mathrm{Ar}), 13.92(2 \mathrm{H}, \mathrm{br}, \mathrm{OH}) . \delta_{\mathrm{C}}\left(125 \mathrm{MHz}\right.$; DMSO-d $\left.\mathrm{d}_{6} \mathrm{Me}_{4} \mathrm{Si}\right) 32.41,120.67,123.62,126.92,128.05$, $128.93,129.45,130.32,135.94,137.36,140.60,141.18$.

4,4'-[(4-Flurophenyl)methylene]bis(3-methyl-1-phenyl-1H-pyrazol-5-ol) (Table 2, entry 9). White solid; mp 181-183 ${ }^{\circ} \mathrm{C}$ [Lit.: (Li et al., 1998) $182{ }^{\circ} \mathrm{C}$ ]; $\delta_{\mathrm{H}}\left(500 \mathrm{MHz}\right.$; DMSO-d 6 ; $\left.\mathrm{Me}_{4} \mathrm{Si}\right) 2.31\left(6 \mathrm{H}, \mathrm{s}, 2 \times \mathrm{CH}_{3}\right), 4.95(1 \mathrm{H}, \mathrm{s}$, $\mathrm{CH}), 7.09(2 \mathrm{H}, \mathrm{t}, J$ 8.8 Ar), 7.22-7.28 (4H, m, Ar), $7.43(4 \mathrm{H}, \mathrm{t}, J 7.7 \mathrm{Ar}), 7.70(4 \mathrm{H}, \mathrm{d}, J 7.9 \mathrm{Ar}), 12.48(1 \mathrm{H}, \mathrm{br}$, $\mathrm{OH}), 13.91(1 \mathrm{H}, \mathrm{s}, \mathrm{OH}) . \delta_{\mathrm{C}}\left(125 \mathrm{MHz}\right.$; DMSO-d $\left.{ }_{6} ; \mathrm{Me}_{4} \mathrm{Si}\right) 12.43,33.32,115.51,115.68,121.42,126.45,129.74$, $129.78,129.85,129.92,139.07,147.11,162.50$.

4,4'-[(4-Nitrophenyl)methylene]bis(3-methyl-1-phenyl-1H-pyrazol-5-ol) (Table 2, entry 10). Yellow solid; mp 228-230 ${ }^{\circ} \mathrm{C}$, [Lit.: (Wang et al., 2005) $230-232{ }^{\circ} \mathrm{C}$ ]; $\delta_{\mathrm{H}}\left(400 \mathrm{MHz}\right.$; DMSO-d ${ }_{6}$; $\mathrm{Me}_{4} \mathrm{Si}$ ) $2.28\left(6 \mathrm{H}, \mathrm{s}, 2 \times \mathrm{CH}_{3}\right.$ ), $5.06(1 \mathrm{H}, \mathrm{s}, \mathrm{CH}), 7.18(2 \mathrm{H}, \mathrm{t}, J 7.1 \mathrm{Ar}), 7.38(4 \mathrm{H}, \mathrm{t}, J 7.31 \mathrm{Ar}), 7.45(2 \mathrm{H}, \mathrm{d}, J 8.3 \mathrm{Ar}), 7.64$ (4H, d, J 7.8 Ar), 8.10 $\left(2 \mathrm{H}, \mathrm{d}, J\right.$ 8.6 Ar), $13.81(2 \mathrm{H}, \mathrm{br}, \mathrm{OH}) . \delta_{\mathrm{C}}\left(100 \mathrm{MHz}\right.$; DMSO-d ${ }_{6}$; $\left.\mathrm{Me}_{4} \mathrm{Si}\right) 34.45,121.91,124.65,127.03,129.92$, 130.25, 147.20, 147.58, 151.63.

4,4'-[(3-Nitrophenyl)methylene]bis(3-methyl-1-phenyl-1H-pyrazol-5-ol) (Table 2, entry 11). Pale yellow 
solid; mp 151-153 ${ }^{\circ} \mathrm{C}$, [Lit.: (Wang et al., 2005) $149-150{ }^{\circ} \mathrm{C}$ ]; $\delta_{\mathrm{H}}\left(400 \mathrm{MHz}\right.$; DMSO-d ${ }_{6}$; $\left.\mathrm{Me}_{4} \mathrm{Si}\right) 2.35(6 \mathrm{H}, \mathrm{s}, 2 \times$ $\left.\mathrm{CH}_{3}\right), 5.14(1 \mathrm{H}, \mathrm{s}, \mathrm{CH}), 7.26(2 \mathrm{H}, \mathrm{t}, J 7.3 \mathrm{Ar}), 7.45(4 \mathrm{H}, \mathrm{t}, J 7.6 \mathrm{Ar}), 7.60(1 \mathrm{H}, \mathrm{t}, J 8.3 \mathrm{Ar}), 7.68-7.74(5 \mathrm{H}, \mathrm{m}, \mathrm{Ar})$, 8.06-8.10 (2H, m, Ar), $13.91(2 \mathrm{H}, \mathrm{br}, \mathrm{OH}) . \delta_{\mathrm{C}}\left(100 \mathrm{MHz}\right.$; DMSO-d $\left.\mathrm{d}_{6} ; \mathrm{Me}_{4} \mathrm{Si}\right) 32.80,120.63,121.21,121.70$, $125.78,125.81,128.98,129.71,134.34,137.39,144.56,146.30,147.72$.

4,4'-[(4-Cyanophenyl)methylene]bis(3-methyl-1-phenyl-1H-pyrazol-5-ol) (Table 2, entry 12). Yellow solid; mp 210-212 ${ }^{\circ} \mathrm{C}$, [Lit.: (Niknam et al., 2010) $210-212{ }^{\circ} \mathrm{C}$ ]; $\delta_{\mathrm{H}}\left(400 \mathrm{MHz}\right.$; DMSO-d 6 ; $\left.\mathrm{Me}_{4} \mathrm{Si}\right) 2.33\left(6 \mathrm{H}, \mathrm{s}, 2 \times \mathrm{CH}_{3}\right.$ ), $5.07(1 \mathrm{H}, \mathrm{s}, \mathrm{CH}), 7.25(2 \mathrm{H}, \mathrm{t}, J 7.3 \mathrm{Ar}), 7.42-7.46(6 \mathrm{H}, \mathrm{m}, \mathrm{Ar}), 7.40(4 \mathrm{H}, \mathrm{d}, J 7.8 \mathrm{Ar}), 7.76(2 \mathrm{H}, \mathrm{d}, J 8.3 \mathrm{Ar})$, $13.89(2 \mathrm{H}, \mathrm{br}, \mathrm{OH}) . \delta_{\mathrm{C}}\left(100 \mathrm{MHz} ; \mathrm{DMSO}-\mathrm{d}_{6} ; \mathrm{Me}_{4} \mathrm{Si}\right) 33.23,119.00,120.61,125.57,128.38,128.94,133.36$, $142.59,148.15$.

4,4'-[(2-Naphthyl)methylene]bis(3-methyl-1-phenyl-1H-pyrazol-5-ol) (Table 2, entry 13). White solid; mp 206-208 ${ }^{\circ} \mathrm{C}$; [Lit.: (Niknam \& Mirzaee, 2011) 206-208 ${ }^{\circ} \mathrm{C}$ ]; $\delta_{\mathrm{H}}\left(500 \mathrm{MHz}\right.$; DMSO-d $\mathrm{d}_{6}$; $\left.\mathrm{Me}_{4} \mathrm{Si}\right) 2.36(6 \mathrm{H}, \mathrm{s}, 2 \times$ $\left.\mathrm{CH}_{3}\right), 5.14(1 \mathrm{H}, \mathrm{s}, \mathrm{CH}), 7.24(2 \mathrm{H}, \mathrm{t}, J 6.9 \mathrm{Ar}), 7.41-7.45(7 \mathrm{H}, \mathrm{m}, \mathrm{Ar}), 7.71-7.73(5 \mathrm{H}, \mathrm{m}, \mathrm{Ar}), 7.81-7.85(3 \mathrm{H}, \mathrm{m}$, Ar), $12.41(1 \mathrm{H}, \mathrm{br}, \mathrm{OH}), 13.93(1 \mathrm{H}, \mathrm{s}, \mathrm{OH}) . \delta_{\mathrm{C}}\left(125 \mathrm{MHz}\right.$; DMSO-d $\mathrm{d}_{6}$; Me $\left.{ }_{4} \mathrm{Si}\right) 12.50,34.22,121.46,125.80$, $126.30,126.83,127.36,128.16,128.52,128.59,129.79,132.54,133.73,140.55,147.19$.

4,4'-[(3-Pyridyl)methylene]bis(3-methyl-1-phenyl-1H-pyrazol-5-ol) (Table 2, entry 14). White cream solid; mp 238-240 ${ }^{\circ} \mathrm{C}$; [Lit.: (Niknam \& Mirzaee, 2011) $238-240{ }^{\circ} \mathrm{C}$ ]; $\delta_{\mathrm{H}}\left(500 \mathrm{MHz}\right.$; DMSO-d ${ }_{6}$; $\left.\mathrm{Me}_{4} \mathrm{Si}\right) 2.34(6 \mathrm{H}, \mathrm{s}, 2$ $\left.\times \mathrm{CH}_{3}\right), 5.05(1 \mathrm{H}, \mathrm{s}, \mathrm{CH}), 7.23(2 \mathrm{H}, \mathrm{t}, J 7.1 \mathrm{Ar}), 7.34(1 \mathrm{H}, \mathrm{t}, J 6.0 \mathrm{Ar}), 7.43(4 \mathrm{H}, \mathrm{t}, J 7.5 \mathrm{Ar}), 7.71-7.73(5 \mathrm{H}, \mathrm{m}$, Ar), $8.41(1 \mathrm{H}, \mathrm{d}, J 3.6 \mathrm{Ar}), 8.51(1 \mathrm{H}, \mathrm{s}, \mathrm{Ar}), 12.10(1 \mathrm{H}, \mathrm{br}, \mathrm{OH}), 14.12(1 \mathrm{H}, \mathrm{br}, \mathrm{OH}) . \delta_{\mathrm{C}}\left(125 \mathrm{MHz}\right.$; DMSO-d $\mathrm{d}_{6}$; $\left.\mathrm{Me}_{4} \mathrm{Si}\right) 12.54,31.96,104.68,121.45,124.22,126.44,129.76,136.16,138.25,138.91,147.04,147.61,149.32$.

4,4'-[(2-Thienyl)methylene]bis(3-methyl-1-phenyl-1H-pyrazol-5-ol) (Table 2, entry 15). White cream solid; mp 181-183 ${ }^{\circ} \mathrm{C}$, [Lit.: (Niknam et al., 2010) $181-183{ }^{\circ} \mathrm{C}$ ]; $\delta_{\mathrm{H}}\left(400 \mathrm{MHz}\right.$; DMSO-d 6 ; $\left.\mathrm{Me}_{4} \mathrm{Si}\right) 2.32\left(6 \mathrm{H}, \mathrm{s}, 2 \times \mathrm{CH}_{3}\right)$, $5.13(1 \mathrm{H}, \mathrm{s}, \mathrm{CH}), 6.75-6.77(1 \mathrm{H}, \mathrm{m}, \mathrm{Ar}), 6.90-6.92(1 \mathrm{H}, \mathrm{m}, \mathrm{Ar}), 7.24-7.30(3 \mathrm{H}, \mathrm{m}, \mathrm{Ar}), 7.45(4 \mathrm{H}, \mathrm{t}, J 7.8 \mathrm{Ar})$, $7.71(4 \mathrm{H}, \mathrm{d}, J 7.82 \mathrm{Ar}) 14.01(2 \mathrm{H}, \mathrm{br}, \mathrm{OH}) . \delta_{\mathrm{C}}(100 \mathrm{MHz}$; DMSO-d 6 ; Me $4 \mathrm{Si}) 29.43,120.58,124.05,124.15$, $126.75,128.94,132.99,134.13,147.73$.

4,4'-(Methylene)bis(3-methyl-1-phenyl-1H-pyrazol-5-ol) (Table 2, entry 16): White cream solid; mp 225-227 ${ }^{\circ} \mathrm{C}$, [Lit.: Wang et al., 2005) $227-229^{\circ} \mathrm{C}$ ]; $\delta_{\mathrm{H}}\left(400 \mathrm{MHz}\right.$; DMSO-d 6 ; $\left.\mathrm{Me}_{4} \mathrm{Si}\right) 2.22\left(6 \mathrm{H}, \mathrm{s}, 2 \times \mathrm{CH}_{3}\right), 3.24(2 \mathrm{H}, \mathrm{s}$, $\left.\mathrm{CH}_{2}\right), 7.18-7.26(2 \mathrm{H}, \mathrm{m}, \mathrm{Ar}), 7.38-7.50(4 \mathrm{H}, \mathrm{m}, \mathrm{Ar}), 7.65-7.75(4 \mathrm{H}, \mathrm{m}, \mathrm{Ar}), 12.00(1 \mathrm{H}, \mathrm{br}, \mathrm{OH}), 13.17(1 \mathrm{H}, \mathrm{br}$, $\mathrm{OH}) . \delta_{\mathrm{C}}\left(100 \mathrm{MHz}\right.$; DMSO-d 6 ; $\left.\mathrm{Me}_{4} \mathrm{Si}\right) 14.69,18.16,120.17,125.69,128.92,136.68,142.41$.

\section{Results}

In continuation of our studies towards the preparation and applications of heterogeneous solid acid catalysts (Niknam et al., 2010; Niknam et al., 2011; Tayebi et al., 2011; Nouri Sefat et al., 2011; Niknam et al., 2010; Niknam \& Saberi, 2009; Niknam et al., 2010; Niknam et al., 2010), herein we wish to report an efficient procedure for the synthesis of 4,4'-(arylmethylene)bis(3-methyl-1-phenyl-pyrazol-5-ols) via condensation reaction between aldehydes and 5-methyl-2-phenyl-2,4-dihydro-3H-pyrazol-3-one in the presence of $[$ Sipmim $] \mathrm{HSO}_{4}$ as solid acid catalyst (Scheme 2).

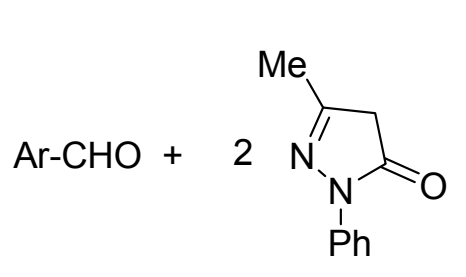

1

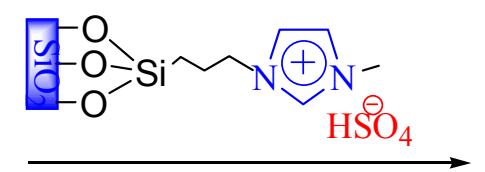

Ethanol, Reflux<smiles>Cc1nn(-c2ccccc2)c(O)c1C([Al])c1c(C)nn(-c2ccccc2)c1O</smiles>

3 a-p

Scheme 2. The condensation of aromatic aldehydes with 3-methyl-1-phenyl-5-pyrazolone catalyzed by $[\mathrm{Sipmim}] \mathrm{HSO}_{4}$ 
The FT-IR spectra of [Sipmim] $\mathrm{HSO}_{4}$ was shown in Figure 1. The IR spectrum shows the overlap asymmetric and symmetric stretching bands of $\mathrm{SO}_{2}$ with $\mathrm{Si}-\mathrm{O}-\mathrm{Si}$ stretching bands in the silica functionalized alkyl-sulfuric acid. The spectrum also shows a broad $\mathrm{OH}$ stretching absorption around 3600 to $2600 \mathrm{~cm}^{-1}$.

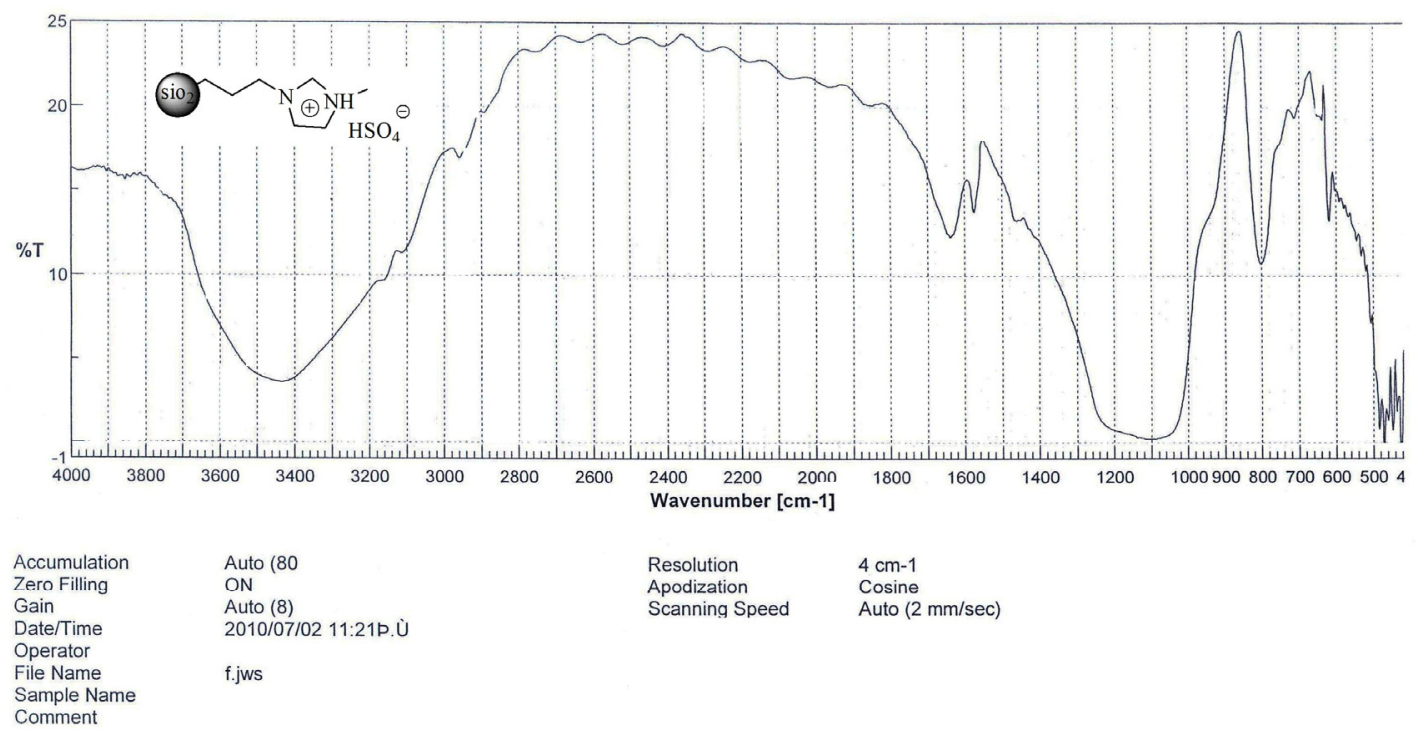

Figure 1. FT-IR of [Sipmim] $\mathrm{HSO}_{4}$

The thermogravimetric analysis (TGA) curve of [Sipmim] $\mathrm{HSO}_{4}$ shows the mass loss of organic materials as they decompose upon heating (Figure 2). The weight loss below $125{ }^{\circ} \mathrm{C}$ corresponds to desorption of physically adsorbed solvent and surface hydroxyl groups. The weight loss contered at higher temperature of about $9 \%$ between 150 and $500{ }^{\circ} \mathrm{C}$ should be attributed to the thermal decomposition of organic groups.

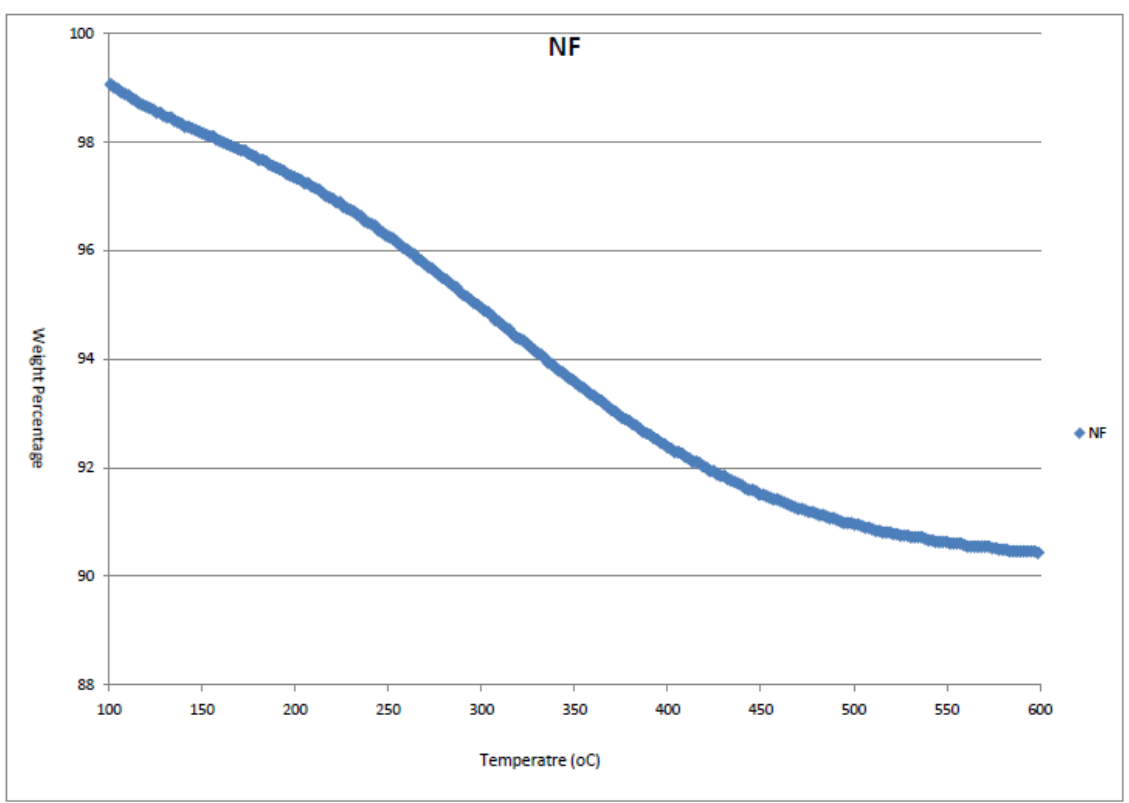

Figure 2. TGA of $\left[\mathrm{Sipmim}_{\mathrm{HSO}} \mathrm{HS}_{4}\right.$

The BET surface area using nitrogen adsorption isotherms at the temperature of liquid nitrogen which gave the results of $\mathrm{a}_{\mathrm{s}, \mathrm{BET}} 1.11 \mathrm{~m}^{2} \mathrm{~g}^{-1}$ and the total pore volume $0.1737 \mathrm{~cm}^{3} \mathrm{~g}^{-1}$ (see supplementary material).

Initial studies were carried out on the condensation reaction between 4-chlorobenzaldehyde and 
3-methyl-1-phenyl-5-pyrazolone in the presence of catalytic amounts of [Sipmim] $\mathrm{HSO}_{4}$ as a model reaction (Table 1). A blank experiment without catalyst gave very low yield after $24 \mathrm{~h}$. The optimal amount of [Sipmim] $\mathrm{HSO}_{4}$ was $0.15 \mathrm{~g}$ (equal to $0.083 \mathrm{mmol}$ of $\mathrm{H}^{+}$) per $1 \mathrm{mmol}$ of aldehyde in refluxing ethanol.

Table 1. Condensation reaction of 4-chlorobenzaldehyde with 5-methyl-2-phenyl-2,4-dihydro-3H-pyrazol-3-one in the presence of different amounts of catalysts ${ }^{\text {a }}$

\begin{tabular}{|c|c|c|c|c|}
\hline Entry & Catalyst & Catalyst loading (g) & Time (min) & Yield $\%^{\mathrm{b}}$ \\
\hline 1 & No catalyst & - & $24 \mathrm{~h}$ & $<10$ \\
\hline 2 & {$[\mathrm{Sipmim}] \mathrm{HSO}_{4}$} & $0.05\left(0.027 \mathrm{mmol}\right.$ of $\left.\mathrm{H}^{+}\right)$ & 130 & 81 \\
\hline 3 & {$[\mathrm{Sipmim}] \mathrm{HSO}_{4}$} & $0.07\left(0.038 \mathrm{mmol}\right.$ of $\left.\mathrm{H}^{+}\right)$ & 90 & 84 \\
\hline 4 & {$[\mathrm{Sipmim}] \mathrm{HSO}_{4}$} & $0.15\left(0.083 \mathrm{mmol}\right.$ of $\left.\mathrm{H}^{+}\right)$ & 60 & 90 \\
\hline 5 & {$[\mathrm{Sipmim}] \mathrm{HSO}_{4}$} & $0.20\left(0.11 \mathrm{mmol}\right.$ of $\left.\mathrm{H}^{+}\right)$ & 60 & 90 \\
\hline
\end{tabular}

${ }^{\mathrm{a}}$ Reaction conditions: 4-chlorobenzaldehyde $(1 \mathrm{mmol})$, 5-methyl-2-phenyl-2,4-dihydro-3H- pyrazol-3-one (2 $\mathrm{mmol})$, ethanol $(6 \mathrm{ml})$ reflux conditions. ${ }^{\mathrm{b}}$ Isolated yield.

Using the optimized conditions, the catalytic efficiency of [Sipmim] $\mathrm{HSO}_{4}$ was also observed for other substituted aromatic aldehydes (Scheme 2 and Table 2).

Table 2. Preparation of 4,4'-(arylmethylene)-bis-(3-methyl-1-phenyl-1H-pyrazol-5-ols) derivatives catalyzed by $[\mathrm{Sipmim}] \mathrm{HSO}_{4}$ in ethanol under refluxing conditions ${ }^{\mathrm{a}}$

\begin{tabular}{|c|c|c|c|c|c|c|}
\hline Entry & $\operatorname{Ar}(1)$ & Product & Time (h) & Yield $\%{ }^{b}$ & $\operatorname{Mp}\left({ }^{\circ} \mathrm{C}\right)$ & Lit. $\mathrm{mp}\left({ }^{\circ} \mathrm{C}\right)$ \\
\hline 1 & $\mathrm{C}_{6} \mathrm{H}_{5^{-}}$ & $\mathbf{3 a}$ & 2.0 & 89 & $170-172$ & 171-172 (Wang et al., 2005) \\
\hline 2 & 4-Me- $\mathrm{C}_{6} \mathrm{H}_{4}-$ & $3 \mathbf{b}$ & 2.2 & 88 & $202-204$ & 203 (Li et al., 1998) \\
\hline 3 & $4-\mathrm{C}_{6} \mathrm{H}_{5} \mathrm{CH}_{2} \mathrm{O}-\mathrm{C}_{6} \mathrm{H}_{4^{-}}$ & $3 c$ & 3.5 & 77 & $210-212$ & 214 (Li et al., 1998) \\
\hline 4 & 4-MeCONH- $\mathrm{C}_{6} \mathrm{H}_{4-}$ & 3d & 4.5 & 86 & $251-253$ & - \\
\hline 5 & $5-\mathrm{Br}-2-(\mathrm{OH})-\mathrm{C}_{6} \mathrm{H}_{3}-$ & $3 e$ & 3.5 & 87 & $266-268$ & - \\
\hline 6 & $2-\mathrm{Br}-\mathrm{C}_{6} \mathrm{H}_{4}^{-}$ & $3 f$ & 2.5 & 81 & $198-200$ & 198-200 (Tayebi et al., 2011) \\
\hline 7 & $4-\mathrm{Cl}-\mathrm{C}_{6} \mathrm{H}_{4}-$ & $3 g$ & 1.0 & 90 & $215-217$ & 210 (Li et al., 1998) \\
\hline 8 & $2-\mathrm{Cl}-\mathrm{C}_{6} \mathrm{H}_{4}-$ & $3 \mathrm{~h}$ & 1.25 & 83 & $235-237$ & 236-237 (Wang et al., 2005) \\
\hline 9 & $4-\mathrm{F}-\mathrm{C}_{6} \mathrm{H}_{4-}^{-}$ & $3 \mathbf{i}$ & 1.15 & 87 & $181-183$ & 182 (Li et al., 1998) \\
\hline 10 & $4-\mathrm{O}_{2} \mathrm{~N}-\mathrm{C}_{6} \mathrm{H}_{4-}^{-}$ & $\mathbf{3 j}$ & 2.0 & 88 & $228-230$ & 230-232 (Wang et al., 2005) \\
\hline 11 & $3-\mathrm{O}_{2} \mathrm{~N}-\mathrm{C}_{6} \mathrm{H}_{4}-$ & $3 \mathbf{k}$ & 1.75 & 89 & $151-153$ & 149-150 (Wang et al., 2005) \\
\hline 12 & 4-(CN)- $\mathrm{C}_{6} \mathrm{H}_{4^{-}}$ & 31 & 1.0 & 87 & $210-212$ & 210-212 (Niknam et al., 2010) \\
\hline 13 & $2-\mathrm{C}_{10} \mathrm{H}_{7^{-}}$ & $3 \mathrm{~m}$ & 3.0 & 90 & $206-208$ & $\begin{array}{l}\text { 206-208 (Niknam \& Mirzaee, } \\
\text { 2011) }\end{array}$ \\
\hline 14 & 3-Pyridyl- & $3 n$ & 3.0 & 82 & $238-240$ & $\begin{array}{l}\text { 238-240 (Niknam \& Mirzaee, } \\
\text { 2011) }\end{array}$ \\
\hline 15 & 2-Thienyl- & 30 & 3.0 & 80 & $181-183$ & 181-183 (Niknam et al., 2010) \\
\hline 16 & H- & $3 p$ & 2.0 & 82 & $225-227$ & 227-229 (Wang et al., 2005) \\
\hline
\end{tabular}

${ }^{\mathrm{a}}$ Reaction conditions: aromatic aldehyde $(1 \mathrm{mmol}), 5$-methyl-2-phenyl-2,4-dihydro- $3 \mathrm{H}$-pyrazol-3-one $(2 \mathrm{mmol})$, catalyst $[\mathrm{Sipmim}] \mathrm{HSO}_{4}(0.15 \mathrm{~g})$, ethanol $(6 \mathrm{~mL})$, reflux conditions. ${ }^{\mathrm{b}}$ Isolated yield.

As shown in Table 2, a series of benzaldehydes including electron-donating or electron-withdrawing groups, i.e. methyl, benzyloxy, and acetamido-benzaldehyde (Table 2, entries 2-4) or 4-nitro, 3-nitro, and 
4-cyano-benzaldehyde (Table 2, entries 10-12), were condensed into the corresponding 4,4'-(arylmethylene)-bis-(3-methyl-1-phenyl- $1 H$-pyrazol-5-ols) $\mathbf{3 b}$-3d and $\mathbf{3 j - 3 \mathbf { l }}$ in very good yields. 2-Naphthyl carbaldehyde was treated with 5-methyl-2-phenyl-2,4-dihydro-3 $H$-pyrazol-3-one gave into corresponding product $\mathbf{3 m}$ in $90 \%$ yield (Table 2, entry 13 ). Heteroaromatic aldehydes such as 3-pyridine carbaldehyde and thiophene-2-carbaldehyde (Table 2, entries 14,15) were reacted with 5-methyl-2-phenyl-2,4-dihydro$3 \mathrm{H}$-pyrazol-3-one gave the corresponding products $3 \mathbf{n}, 3 \mathbf{3 o}$ in $82 \%$ and $80 \%$ yield respectively. Formaldehyde was converted into the corresponding product $3 \mathbf{p}$ in $82 \%$ yield (Table 2 , entry 16 ).

The condensation reaction between 4-chlorobenzaldehyde and 5-methyl-2-phenyl-2,4-dihydro-3 $H$-pyrazol-3-one was examined for the possibility of recycling [Sipmim] $\mathrm{HSO}_{4}$ under the optimized conditions. After completion, the reaction mixture was washed with warm ethanol $(3 \times 30 \mathrm{~mL})$. The recovered catalyst was washed with diethyl ether, dried and reused for subsequent runs. [Sipmim] $\mathrm{HSO}_{4}$ showed much the same efficiency when used in six consecutive reactions runs (Figure 3).

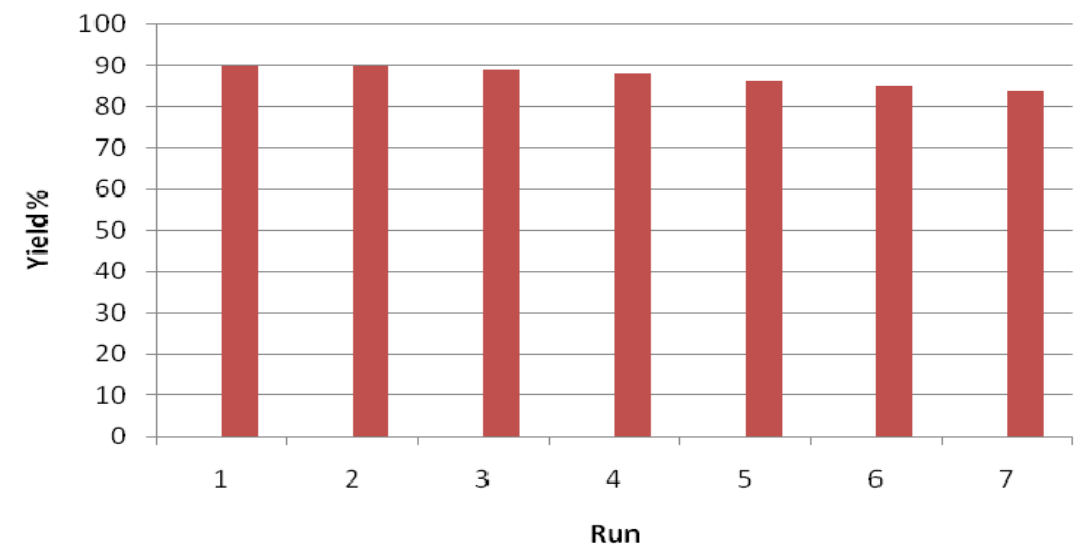

Figure 3. Recyclability of [Sipmim] $\mathrm{HSO}_{4}$ as catalyst in the condensation reaction of 4-chlorobenzaldehyde (1 $\mathrm{mmol})$ and 5-methyl-2-phenyl-2,4-dihydro-3 $\mathrm{H}$-pyrazol-3-one $(2 \mathrm{mmol})$ in the presence of $0.15 \mathrm{~g}$ of

$[\mathrm{Sipmim}] \mathrm{HSO}_{4}$ in refluxing ethanol. Reaction time $=1.0 \mathrm{~h}$

In conclusion, the synthesis of bis-pyrazolones via three-component condensation reaction of aldehydes with two equivalents of 5-methyl-2-phenyl-2,4-dihydro-3 $\mathrm{H}$-pyrazol-3-one using [Sipmim] $\mathrm{HSO}_{4}$ as heterogeneous acid catalyst in refluxing ethanol, and also recovery and reusability of the catalyst, high yields, and clean work-up makes this method practical.

\section{Acknowledgements}

Financial support for this work by the Research Council of Islamic Azad University, Gachsaran Branch, Iran, is gratefully acknowledged.

\section{References}

Bailey, D. M., Hansen, P. E., Hlavac, A. G., Baizman, E. R., Pearl, J., Defelice, A. F., \& Feigenson, M. E. (1985). 3,4-Diphenyl-1H-pyrazole-1-propanamine antidepressants. J. Med. Chem., 28(2), 256-260. http://dx.doi.org/10.1021/jm00380a020

Buzykin, B. I., \& Lonshchakova, T. I. (1971). Reaction of azines with 5-pyrazolones. Bull. Acad. Sci. USSR, Div. Chem. Sci. (Engl. Trans.), 20(10), 2224-2226. http://dx.doi.org/10.1007/BF00851295

Chauhan, P. M. S., Singh, S., \& Chatterjee, R. K. (1993). Antifilarial Profile of Substituted Pyrazoles: A New Class of Antifilarial Agents. Indian J. Chem., Sect. B, 32, 858-861. http://dx.doi.org/10.1002/chin.199347186

Cho, I. H., Noh, J. Y., Park, S. W., Ryu, H. C., Lim, J. W., Kim, J. H., Chae, M. Y., Kim, D. H., Jung, S. H., Park, H. J., Kim, Y. H., \& Min, I. K. (2004). US Patent, 2,004, 002, 532.

Chrobok, A., Baj, S., Pudlo, W., \& Jarzebski, A. (2009). Supported hydrogensulfate ionic liquid catalysis in Baeyer-Villiger reaction. Appl. Catal. A: Gen., 366(1), 22-28. http://dx.doi.org/10.1016/j.apcata.2009.06.040

Clark, M. P., Laughlin, S. K., Golebiowski, A., Brugel, T. A., Sabat, M. (2005). WO Patent, 2, 005, 047, 287. 
Clark, M. P., Laughlin, S. K., Laufersweiler, M. J., Bookland, R. G., Brugel, T. A., Golebiowski, A., Sabat, M. P. et al. (2004). Development of Orally Bioavailable Bicyclic Pyrazolones as Inhibitors of Tumor Necrosis Factor- $\alpha$ Production. J. Med. Chem., 47(11), 2724-2727. http://dx.doi.org/10.1021/jm049968m

Elinson, M. N., Dorofeev, A. S., Nasybullin, R. F., \& Nikishin, G. I. (2008). Facile and Convenient Synthesis of 4,4'-(Arylmethylene)bis(1H-pyrazol-5-ols) by Electrocatalytic Tandem Knoevenagel-Michael Reaction. Synthesis, 2008(12), 1933-1937. http://dx.doi.org/10.1055/s-2008-1067079

Gupta, N., Sonu, G. L., Kad, \& Singh, J. (2007). Acidic ionic liquid [bmim] $\mathrm{HSO}_{4}$ : An efficient catalyst for acetalization and thioacetalization of carbonyl compounds and their subsequent deprotection. Catal. Commun., 8(9), 1323-1328. http://dx.doi.org/10.1016/j.catcom.2006.11.030

Hajipour, A. R., Rajaei, A., \& Ruoho, A. E. (2009). A mild and efficient method for preparation of azides from alcohols using acidic ionic liquid [H-NMP]HSO ${ }_{4}$. Tetrahedron Lett., 50(6), 708-711. http://dx.doi.org/10.1016/j.tetlet.2008.11.111

Hamama, W. S. (2001). Pyrazolones as versatile precursors for the synthesis of fused and binary heterocycles. Synth. Commun., 31(9), 1335-1345. http://dx.doi.org/10.1081/SCC-100104042

Keim, W., Korth, W., \& Wasserscheid, P. (2000). WO 016,902 Al, March 30.

Li, X. L., Wang, Y. M., Tian, B., Matsuura, T., \& Meng, J. B. (1998). The solid-state michael addition of $\begin{array}{lllll}\text { 3-methyl-1-phenyl-5-pyrazolone. } \quad J . & \text { Heterocycl. }\end{array}$ http://dx.doi.org/10.1002/jhet.5570350124

Londershausen, M. (1996). Review: Approaches to New Parasiticides. Pestic. Sci., 48(4), 269-292. http://dx.doi.org/10.1002/(SICI)1096-9063(199612)

Mahajan, R. N., Havaldar, F. H., \& Fernandes, P. S. (1991). Syntheses and biological activity of heterocycles derived from 3-methoxy-1-phenyl-1H-pyrazole-5-carboxylate. J. Indian Chem. Soc., 68, 245-246.

Mosaddegh, E., Hassankhani, A., \& Baghizadeh, A. (2010). Cellulose Sulfuric Acid as a New Biodegradable and environmetally Friendly Biopolymer for Synthesis of 4,4'-(arylmethylene)bis(3-methyl-1-phenyl-1H-pyrazol-5-ols). J. Chil. Chem. Soc., 55(4), 419-420. http://dx.doi.org/10.4067/S0717-9707201000040000

Niknam, K., \& Damya, M. (2009). 1-Butyl-3-methylimidazolium Hydrogen Sulfate [bmim]HSO4: An Efficient Reusable Acidic Ionic Liquid for the Synthesis of 1,8-Dioxo-octahydroxanthenes. J. Chin. Chem. Soc., 56(3), 659-665.

Niknam, K., Deris, A., Naeimi, F., \& Majleci, F. (2011). Synthesis of 1,2,4,5-tetrasubstituted imidazoles using silica-bonded propylpiperazine N-sulfamic acid as a recyclable solid acid catalyst. Tetrahedron Lett., 52(36), 4642-4645. http://dx.doi.org/10.1016/j.tetlet.2011.06.105

Niknam, K., Mohammadizadeh, M. R., Mirzaee, S., \& Saberi, D. (2010). Silica-bonded S-Sulfonic Acid: A Recyclable Catalyst for the Synthesis of Trisubstituted Imidazoles under Solvent-free Conditions. Chin. J. Chem., 28(4), 663-669. http://dx.doi.org/10.1002/cjoc.201090129

Niknam, K., \& Mirzaee, S. (2011). Silica Sulfuric Acid an Efficient and Recyclable Solid Acid Catalyst for the Synthesis of 4,4'-(Arylmethylene)bis(1H-pyrazol-5-ols). Synth. Commun., 41(16), 2403-2413. http://dx.doi.org/10.1080/00397911.2010.502999

Niknam, K., \& Saberi, D. (2009). Preparation of sulfuric acid ([3-(3-silicapropyl)sulfanyl]propyl)ester: A new and recyclable catalyst for the formylation and acetylation of alcohols under heterogeneous conditions. Appl. Catal. A: Gen., 366(1-2), 220-225. http://dx.doi.org/10.1016/j.apcata.2009.07.014

Niknam, K., Saberi, D., \& Nouri Sefat, M. (2010). Silica-bonded S-sulfonic acid: an efficient and recyclable solid acid catalyst for the three-component synthesis of a-amino nitriles. Tetrahedron Lett., 51(22), 2959-2962. http://dx.doi.org/10.1016/j.tetlet.2010.03.093

Niknam, K., Saberi, D., Sadegheyan, M., \& Deris, A. (2010). Silica-bonded S-sulfonic acid: an efficient and recyclable solid acid catalyst for the synthesis of 4,4'-(arylmethylene)bis(1H-pyrazol-5-ols). Tetrahedron Lett., 51(4), 692-694. http://dx.doi.org/10.1016/j.tetlet.2009.11.114.

Niknam, K., Zolfigol, M. A., Saberi, D., \& Khonbazi, M. (2009). 1-Butyl-3-methylimidazolium Hydrogen Sulfate [bmim] $\mathrm{HSO}_{4}$ : An Efficient Reusable Acidic Ionic Liquid for the Formylation of Alcohols. Chin. J. Chem., 27(8), 1548-1552. http://dx.doi.org/10.1002/cjoc.200990261

Nouri Sefat, M., Saberi, D., \& Niknam, K. (2011). Preparation of Silica-Based Ionic Liquid an Efficient and Recyclable Catalyst for One-Pot Synthesis of a-Aminonitriles. Catal. Lett., 141(11), 1713-1720. 
http://dx.doi.org/10.1007/s10562-011-0696-x

Park, H. J., Kim, Y. H., \& Min, I. K. (2004). US Patent, 2,004, 002, 532.

Park, H. J., Lee, K., Park, S. J., Ahn, B., Lee, J. C., Cho, H. Y., \& Lee, K. I. (2005). Identification of antitumor activity of pyrazole oxime ethers. Bioorg. Med. Chem. Lett., 15(13), 3307-3312. http://dx.doi.org/10.1016/j.bmcl.2005.03.082

Pavlov, P. T., Goleneva, A. F., Lesnov, A. E., \& Prokhorova, T. S. (1998). Biological activity of some pyrazolone derivatives. Pharm. Chem. J. (Engl. Trans.), 32(7), 370-372. http://dx.doi.org/10.1007/BF02645994

Qiao, K., Hagiwara, H., \& Yokoyama, C. (2006). Acidic ionic liquid modified silica gel as novel solid catalysts for esterification and nitration reactions. J. Mol. Catal. A: Chem., 246(1-2), 65-69. http://dx.doi.org/10.1016/j.molcata.2005.07.031

Rosiere, C. E., \& Grossman, M. I. (1951). An Analog of Histamine that Stimulates Gastric Acid Secretion without other Actions of Histamine. Science, 113, 651-651. http://dx.doi.org/10.1126/science.113.2945.651

Singh, D., \& Singh, D. (1984). Syntheses of 1,3-disubstituted 4-arylidenepyrazolin-5-ones and the keto and enol forms of 4,4'-arylidenebis(1,3-disubstituted pyrazolin-5-ones). J. Chem. Eng. Data, 29(3), 355-356. http://dx.doi.org/10.1021/je00037a040

Singh, D., \& Singh, D. (1991). Synthesis and antifungal activity of some 4-arylmetylene derivatives of substituted pyrazolones. J. Indian Chem. Soc., 68, 165-167.

Sugimura, R., Qiao, K., Tomida, D., \& Yokoyama, C. (2007). Immobilization of acidic ionic liquids by copolymerization with styrene and their catalytic use for acetal formation. Catal. Commun., 8(5), 770-772. http://dx.doi.org/10.1016/j.catcom.2006.08.049

Sujatha, K., Shanthi, G., Selvam, N. P., Manoharan, S., Perumal, P. T., \& Rajendran, M. (2009). Synthesis and antiviral activity of 4,4'-(arylmethylene)bis( $1 H$-pyrazol-5-ols) against peste des petits ruminant virus (PPRV). Bioorg. Med. Chem. Lett., 19(15), 4501-4503. http://dx.doi.org/10.1016/j.bmcl.2009.02.113

Tajik, H., Niknam, K., \& Parsa, F. (2009). Using Acidic Ionic Liquid 1-Butyl-3-methylimidazolium Hydrogen Sulfate in Selective Nitration of Phenols under Mild Conditions. J. Iran. Chem. Soc., 6(1), 159-164.

Tajik, H., Niknam, K., \& Sarrafan, M. (2011). 1-butyl-3-methylimidazolium hydrogen sulfate ([bmim]-HSO ${ }_{4}$ )-mediated synthesis of polysubstituted quinolines. Synth. Commun., 41(14), 2103-2114. http://dx.doi.org/10.1080/00397911.2010.497596

Tayebi, S., Baghernejad, M., Saberi, D., \& Niknam, K. (2011). Sulfuric Acid ([3-(3-Silicapropyl)sulfanyl]propyl)ester as a Recyclable Catalyst for the Synthesis of 4,4'-(Arylmethylene)bis(1H-pyrazol-5-ols). Chin. J. Catal., $32(9), \quad$ 1477-1483. http://dx.doi.org/10.1016/S1872-2067(10)60260-4

Wang, W., Cheng, W., Shao, L., \& Yang, J. (2008). [TMBSA][HSO ${ }_{4}$ Ionic Liquid as Novel Catalyst for the Rapid Acetylation of Alcohols, Hydroxyesters and Phenols under Solvent-free Conditions. Catal. Lett., 121(1-2), 77-80. http://dx.doi.org/10.1007/s10562-007-9295-2

Wang, W., Wang, S. X., Qin, X. Y., \& Li, J. T. (2005). Reaction of Aldehydes and Pyrazolones in the Presence of Sodium Dodecyl Sulfate in Aqueous Media. Synth. Commun., 35(9), 1263-1269. http://dx.doi.org/10.1081/SCC-200054854

Wasserscheid, P., Sesing, M., \& Korth, W. (2002). Hydrogensulfate and tetrakis(hydrogensulfato)borate ionic liquids: synthesis and catalytic application in highly Brønsted-acidic systems for Friedel-Crafts alkylation. Green Chem., 4(2), 134-138. http://dx.doi.org/10.1039/b109845b

Wasserscheid, P., \& Welton, T. (2007). Ionic Liquids in Synthesis (2nd ed.). Wiley-VCH, Weinheim. http://dx.doi.org/10.1002/9783527621194

Xu, D. Q., Yang, W. L., Luo, S. P., Wang, B. T., Wu, M., \& Xu, Z. Y. (2007). Fischer Indole Synthesis in Brønsted Acidic Ionic Liquids: A Green, Mild, and Regiospecific Reaction System. Eur. J. Org. Chem., 2007(6), 1007-1012. DOI: 10.1002/ejoc.200600886.

Zang, H., Su, Q., Guo, S., Mo, Y., \& Cheng, B. (2011). An Efficient One-pot Synthesis of Pyrazolone Derivatives Promoted by Acidic Ionic Liquid. Chin. J. Chem., 29(10), 2202-2204. http://dx.doi.org/10.1002/cjoc.201180381 